\title{
Second Class Functional Treatment: Andreasen Activator vs Twin Block
}

\author{
Giulia Baccaglione ${ }^{1}$, Elisa Rota ${ }^{2}$, Maurizio Ferrari ${ }^{3}$, Marcello Maddalone $^{4}$
}

\begin{abstract}
Aim: The purpose of this research is to analyze and compare the dental and skeletal changes induced by two functional devices, Andreasen Activator and Clark's twin block, on the sagittal and vertical plane, by means of cephalometric analysis, of the lateral cephalograms prescribed at the beginning and at the end of the treatment for a second skeletal class, first division with normal or deep bite.

Materials and methods: Twenty patients, 8 females and 12 males, fulfilling criteria for inclusion, were divided randomly into two groups: group I was treated with Andreasen activator, the second group with Clark's twin block. The duration of the therapy was about 18 months plus less 2 months. Pretreatment and posttreatment cephalometric radiographs were analyzed using angular (SNA, SNB, ANB, SnaSnp-GoGn angles), linear (Sna-Snp, Co-Gn, Co-Go, Go-Gn) skeletal parameters and dental one (U1-SnaSnp angle, L1-GoGn angle, Overjet and Overbite). To evaluate the posttreatment changes in the single groups and between them, paired and unpaired $t$-test was used.

Results: In both of the two groups analyzed, all the sagittal and vertical, angular and linear, skeletal measurements appear to be increased in a statistically significant way, except SNA angle and the distance Sna-Snp. Regarding the dental parameters, in the group treated with Andreasen activator, only Overjet and Overbite showed statistically significant differences. On the other hand, twin block induced statistical changes about Overjet, Overbite and also U1/SnaSnp, but not about L1/GoGn. The advancement of the mandible determines a greater prominence of the chin and lower lip, an increment of the labial mental angle and a reduction of the convexity of the profile. Also, the decrease of the overjet and, consequently, of the dental exposure improve the esthetic appearance of the patient's face.

Conclusion: Both functional treatments showed a lower jaw advanced on the sagittal plane and increased in size. In the upper jaw no significant changes were observed. It was also evident a dental compensation both on sagittal and vertical planes.

Clinical significance: The functional devices studied, Andreasen activator and twin block, seem to obtain more skeletal than dental results when the patients were treated at the peak of pubertal growth.

Keywords: Andreasen activator, Cephalometrics, II class, Malocclusion, Mandible, Prospective longitudinal clinical study, Twin block. International Journal of Clinical Pediatric Dentistry (2020): 10.5005/jp-journals-10005-1725
\end{abstract}

\section{INTRODUCTION}

Today orthodontic and dental researches are mostly oriented to use new radiographic analysis or to find and test new treatment devices. ${ }^{1-8}$ Functional appliances have been used for many years in the treatment of Class II malocclusions with the aim to obtain a skeletal correction of malocclusions. In fact, the use of these devices should support improvements in mandibular position and growth together with changes in dental and muscular relationships.

Skeletal Class II malocclusion results from either maxillary protrusion, mandibular retrusion, or a combination of the two..$^{9-11}$

In cases of patients in a growing phase with skeletal Class II malocclusion with mandible retrusion, the Class II Twin block appliance or other mandibular activators can be used to stimulate and enhance mandibular growth.

This can be achieved, first of all, solving skeletal and functional alterations of the whole cranial facial complex, and, only in a second step, with the correction of the position of the individual dental elements.

Many authors have pointed out that growth is not only genetically determined but also related to the environmental situations that can influence direction and range of the growth. ${ }^{12,13}$

The convex facial profile, not particularly harmonious, the increased overjet, the mandibular incisors often hidden by the lower lip can lead the adolescent to be disappointed with his appearance and consequently have a reduced self-esteem.

\footnotetext{
1,4 Department of Medicine and Surgery, University of Milano Bicocca, Monza, Italy

2,3 Department of Orthodontics, University of Milano Bicocca, Monza, Italy
}

Corresponding Author: Giulia Baccaglione, Department of Medicine and Surgery, University of Milano Bicocca, Monza, Italy, Phone: +390392339888, e-mail: giulia.baccaglione@gmail.com

How to cite this article: Baccaglione G, Rota E, Ferrari M, et al. Second Class Functional Treatment: Andreasen Activator vs Twin Block. Int J Clin Pediatr Dent 2020;13(2):144-149.

Source of support: Nil

Conflict of interest: None

In order to intercept the divergent pattern of growth the orthodontist can treat a second class with the use of an orthopedic functional therapy with a mandibular activator. In growing subjects in fact it is important to intercept a wrong model of growth and eliminate it with the aim to restore harmonious dentoskeletal relationships and a pleasant facial profile.

This study is intended to support the use of functional appliances in the correction of second skeletal classes. The functional devices chosen in this study were Andreasen activator and Clark's twin block. Andreasen activator, still widely used today, is one of the first functional devices created in order to stimulate mandibular growth. It was useful in producing good skeletal effects even if it presented a disadvantage: because the upper 
and lower part of the device are joined, the patient cannot have a normal phonetic and functional activity while wearing it. ${ }^{14}$ This reduces the time of use of the device and discourage the patient's collaboration. Therefore, over the years, a great effort has been made to improve the design of the functional appliances to make it possible to use them almost all the day. The Twin block appliance, developed by Clark, is one of the most widely used functional appliances for the correction of Class II skeletal malocclusions. ${ }^{15}$ It consists of coordinated intraoral splints, sometimes combined with an extraoral force, that generate a functional propelling orthopedic force. The field of its common use, as applied in our present study, is the second skeletal class originated by mandibular retrusion. The appliance is usually used most of the time, offering high patient acceptability and ability to produce rapid appreciable results. Furthermore, patients preserve an acceptable speech and a nearly a full range of mandibular movement.

Twin block is a technique that maximizes the growth response to a functional mandibular protrusion through a simple, comfortable and esthetically acceptable device for the patient. In the literature multiple studies highlight the excellent results produced by it in the correction of class II malocclusion. ${ }^{16}$

The aim of this research is to evaluate and compare the efficacy of two functional devices, Andresen's activator and Clark's twin block, in the treatment of the second skeletal class, first division with a normal or deep bite. The study wants to analyze the dental and skeletal changes induced by both devices on the sagittal and vertical plane, by means of cephalometric analysis, of the lateral cephalograms prescribed at the beginning (TO) and at the end of the treatment (T1).

\section{Materials and Methods}

Twenty patients from the Dental Clinic of S. Gerardo Hospital (University of Medicine Milano Bicocca, Monza, Italy) with a diagnosis of II skeletal and dental class in two year time span (April 2015-April 2017) were enrolled to participate in this study. The 20 patients, 8 females and 12 males, were aged between 11 years and 14 years (average age 12.7).

Informed written consent to the treatment and processing of personal data was signed to the parents.

Before starting the treatment, general patient's medical history was collected and physical examination performed.

During the clinical dental examination, intraoral and extraoral photos were taken, radiographic examinations (orthopantomography and lateral cephalograms) and dental casts were collected.

Patients presented clinical characteristics compatible with the study (i.e., second skeletal class / absence of systemic diseases). The lateral cephalograms of all the selected patients had to show a vertebral maturation in-between CS2 and CS3 stages before the beginning of the therapy (T0). ${ }^{17}$

The patients were randomly divided into two groups: group I was treated with the Andreasen activator, the second group with the Clark's twin block. The duration of the therapy was about 18 months plus less 2 months. An objective evaluation of the patient's collaboration was not considered in the study.

Inclusion criteria for this study were:

- Skeletal maturation phase between CS2 and CS3, according to the evaluation method of the cervical vertebrae of Franchi, Baccetti and McNamara ${ }^{17}$

- Bilateral class II incisor, canine and molar relationship
- Skeletal class II relationship caused by mandibular retrusion and normal or decreased facial divergence. The following reference cephalometric values are used:

$$
\text { - SNA. }=80^{\circ} \pm 2^{\circ}
$$

- $\mathrm{SNB}<78^{\circ}$

- Overjet $>4 \mathrm{~mm}$

- $\mathrm{SN}-\mathrm{GoGn} \leq 37^{\circ}$

- Gonial angle (Co-Go-Me) $<128^{\circ}$

- No previous orthodontic treatment or functional therapy in progress

- Absence of dental anomalies (agenesis, supernumerary, included elements)

- Good oral health condition

- Absence of systemic diseases or congenital deformities.

Two lateral cephalograms were obtained, one at the beginning and at the end of the therapy. Cephalometric tracing was performed, by the same operator, using both angular and linear measurements. The evaluated angular skeletal parameters are: SNA, SNB, ANB, SnaSnp-GoGn angles. The distance Sna-Snp, was considered as length of the maxilla, Co-Gn as total mandibular length, $\mathrm{Co}-\mathrm{Go}$ as mandibular ramus height and Go-Gn for mandibular body length, was then evaluated. Finally, dental parameters were assessed: U1SnaSnp angle, L1-GoGn angle, Overjet and Overbite.

Statistical analysis was performed with the aim of Prism 7.0 software (GraphPad Software, Inc.). All the data, through the D'Agostino Pearson test, showed a normal distribution. Statistical analysis was initially performed to compare the measurements between $\mathrm{T} 0$ and $\mathrm{T} 1$ of each study group, in order to verify that the difference in results between the analyzed parameters was actually significant: the paired $t$-test with statistical threshold value was used of $p<0.05$. To evaluate if one of the two devices had, in a statistically significant way, more results than the other unpaired $t$-test was used and the significance of the changes was always tested with $p<0.05$

The study was conducted according to the Declaration of Helsinki (1964, revision 2008) and approved by the local Ethical Committee of the San Gerardo Hospital, University of Milano Bicocca.

\section{Results}

We first took into account the group treated with Andreasen activator (Table 1): with respect to the angular skeletal parameters, the most significant change $(p<0.0001)$ regarded SnaSnp-GoGn (average $\mathrm{T} 0=19.6^{\circ}$, average $\mathrm{T} 1=22.2^{\circ}$ ). Statistically significant changes were also found in the SNB angle $(p<0.001)$ (mean T0 $=$ $77.2^{\circ}$; mean $\left.\mathrm{T} 1=79.3^{\circ}\right)$ and $\mathrm{ANB}(p<0.01)$ (mean T0 $=4,8$ th, average $\mathrm{T} 1=2.5^{\circ}$ ). The SNA angle, on the other hand, was not statistically significant. Within the linear skeletal parameters analyzed, the only one nonstatistically significant was Sna-Snp. At the mandibular level, the most significant data $(p<0.01)$ were Co-Gn and Co-Go. The first value showed an average increase of $5.5 \mathrm{~mm}$ (average $\mathrm{T} 0=$ 102.8; average $\mathrm{T} 1=108.3$ ), the second one undergone an average gain of $3.5 \mathrm{~mm}$ (average T0 =53.2; mean T1 = 56.7). The Go-Gn distance, which is also statistically significant $(p<0.05)$, varied from $66.8 \mathrm{~mm}$ (mean T0) to $70.7 \mathrm{~mm}$ (mean T1). Dental parameters showed statistically significant differences for Ovj $(p<0.05)$ and for $\operatorname{Ovb}(p<0.01)$ : they both had an average reduction of $1.6 \mathrm{~mm}$ at T1. On the other hand, no statistically significant changes were registered for U1/SnaSnp and L1/GoGn: both were reduced on average by $0.4^{\circ}$ at the end of therapy. 
Second Class Functional Treatment: Andreasen Activator vs Twin Block

Table 1: Results of the statistical analysis with paired $t$-test on cephalometric values of patients treated with Andreasen activator

\begin{tabular}{|c|c|c|c|c|c|c|c|}
\hline \multicolumn{8}{|c|}{ Andreasen T1-T0 } \\
\hline & Mean TO & $S D$ & Mean T1 & $S D$ & Difference & $p$ value & Significance \\
\hline SNA $\left(^{\circ}\right)$ & 82.0 & 1.080 & 81.8 & 1.099 & -0.2 & 0.8618 & NS \\
\hline SNB $\left(^{\circ}\right)$ & 77.2 & 1.398 & 79.3 & 1.229 & 2.1 & 0.0006 & $* * *$ \\
\hline ANB $\left(^{\circ}\right)$ & 4.8 & 1.418 & 2.5 & 0.8165 & -2.3 & 0.0016 & $* *$ \\
\hline SnaSnp-GoGn $\left(^{\circ}\right)$ & 19.6 & 3.026 & 22.2 & 3.048 & 2.6 & $<0.0001$ & $* * * *$ \\
\hline Sna-Snp (mm) & 49.7 & 2.83 & 51.8 & 3.521 & 2.1 & 0.0713 & NS \\
\hline Co-Gn (mm) & 102.8 & 3.457 & 108.3 & 6.037 & 5.5 & 0.0016 & $* *$ \\
\hline Co-Go (mm) & 53.2 & 3.190 & 56.7 & 4.595 & 3.5 & 0.0049 & $* *$ \\
\hline Go-Gn (mm) & 66.8 & 3.823 & 70.7 & 5.417 & 3.9 & 0.0342 & * \\
\hline U1/SnaSnp $\left(^{\circ}\right)$ & 115.3 & 5.314 & 114.9 & 6.574 & -0.4 & 0.7473 & NS \\
\hline L1/GoGn $\left(^{\circ}\right)$ & 100.1 & 4.533 & 99.7 & 3.622 & -0.4 & 0.6476 & NS \\
\hline Ovj (mm) & 4.7 & 1.16 & 3.1 & 1.287 & -1.6 & 0.0133 & * \\
\hline Ovb (mm) & 4.3 & 1.398 & 2.7 & 0.948 & -1.6 & 0.0046 & $* *$ \\
\hline
\end{tabular}

${ }^{*} p<0.05 ;{ }^{* *} p<0.01 ;{ }^{* * *} p<0.001 ;{ }^{* * * *} p<0.0001 ; \mathrm{NS}$, not significant

Table 2: Results of the statistical analysis with paired $t$-test on cephalometric values of patients treated with twin block appliance

\begin{tabular}{|c|c|c|c|c|c|c|c|}
\hline \multicolumn{8}{|c|}{ Twin block T1-T0 } \\
\hline & Mean T0 & $S D$ & Mean T1 & $S D$ & Difference & $p$ value & Significance \\
\hline SNA $\left(^{\circ}\right)$ & 81.2 & 1.65 & 81 & 1.247 & -0.2 & 0.0522 & NS \\
\hline $\operatorname{SNB}\left({ }^{\circ}\right)$ & 76.2 & 2.058 & 78.5 & 1.814 & 2.3 & $<0.0001$ & $* * * *$ \\
\hline ANB $\left(^{\circ}\right)$ & 5.1 & 0.9189 & 2.5 & 0.7888 & -2.6 & $<0.0001$ & $* * * *$ \\
\hline SnaSnp-GoGn $\left(^{\circ}\right)$ & 21.1 & 2.079 & 23.1 & 2.331 & 2 & 0.0002 & $* * *$ \\
\hline Sna-Snp (mm) & 52.2 & 2.201 & 53.9 & 1.269 & 1.7 & 0.0526 & NS \\
\hline Co-Gn (mm) & 102.3 & 2.983 & 108.2 & 3.706 & 5.9 & 0.0002 & $* * *$ \\
\hline Co-Go (mm) & 50.7 & 3.268 & 55 & 3.916 & 4.3 & 0.0016 & $* *$ \\
\hline Go-Gn (mm) & 65.9 & 3.348 & 69.6 & 4.858 & 3.7 & 0.0132 & * \\
\hline U1/SnaSnp $\left(^{\circ}\right)$ & 116.1 & 3.414 & 112.4 & 2.875 & -3.7 & 0.0078 & $* *$ \\
\hline L1/GoGn $\left(^{\circ}\right)$ & 99.2 & 2.098 & 100.4 & 2.633 & 1.2 & 0.3009 & NS \\
\hline Ovj $(\mathrm{mm})$ & 6.2 & 2.3 & 3.5 & 0.971 & -2.7 & 0.0039 & $* *$ \\
\hline Ovb (mm) & 4.7 & 2.111 & 2.9 & 0.994 & -1.8 & 0.0120 & * \\
\hline
\end{tabular}

${ }^{*} p<0.05 ;{ }^{* *} p<0.01 ;{ }^{* * *} p<0.001 ;{ }^{* * * *} p<0.0001 ; \mathrm{NS}$, not significant

Patients treated with twin block (Table 2) showed more statistically significant changes $(p<0.0001)$ on the SNB and ANB angle: the first one increased on average by $2.3^{\circ}$ (mean T0 $=76.2^{\circ}$; mean $\mathrm{T} 1=78,5^{\circ}$ ); the second one has reduced on average by $2.6^{\circ}$ (average $\mathrm{T} 0=5.1^{\circ}$, average $\mathrm{T} 1=2.5^{\circ}$ ). Statistically significant changes $(p<0.001)$ were also detected with regard to the SnaSnp-GoGn angle: at the end of the therapy there was an average increase equal to $2^{\circ}$. Instead, the SNA angle did not show statistically significant changes (mean difference between T0 and T1 of $-0.2^{\circ}$ ). As for the linear skeletal parameters analyzed, only Sna-Snp doesn't undergo any statistically significant changes (mean difference between T0 and $\mathrm{T} 1$ of $+1.7 \mathrm{~mm}$ ). All the data concerning the jaw are statistically significant. The most significant change $(p<0.001)$ affected Co-Gn: in fact, it changed from an average before treatment of $102.3 \mathrm{~mm}$ to a $108.2 \mathrm{~mm}$ at the end of therapy (average increase of $5.9 \mathrm{~mm})$. Statistically significant changes were detected with Co-Go $(p<0.01)$ and Go-Gn $(p<0.05)$. The first has undergone an average increase at the end of the treatment of $4.3 \mathrm{~mm}$ (mean $\mathrm{T} 0=50.7$; mean $\mathrm{T} 1=55$ ). The second increased on average by 3.7 $\mathrm{mm}$ (mean $\mathrm{T} 0=65.9 ;$ mean $\mathrm{T} 1=69.6)$. Finally, with regard to the analyzed dental parameters, Ovj $(p<0.01)$ and Ovb $(p<0.05)$ are statistically significant: at the end of therapy, the first presents an average reduction of $1.4 \mathrm{~mm}$, the second of $1.8 \mathrm{~mm}$. The other statistically significant data $(p<0.01)$ was U1/ SnaSnp (difference equal to $-3.7^{\circ}$ ). L1/ GoGn has undergone an average increase, at the end of the treatment, of $1.2^{\circ}$ : it does not result, however, to be statistically significant.

The comparison between the group treated with Andreasen activator and the one with twin block (Table 3) showed that both the studied devices gave the same effectiveness with respect to the SNA parameter. The twin block has given greater efficiency with regard to SNB $\left(+2.3^{\circ}\right.$ compared to $+2.1^{\circ}$ of the activator) and ANB ( $-2.6^{\circ}$ compared to $-2.1^{\circ}$ of the activator). The Co-Gun parameter has increased by more than $+0.4 \mathrm{~mm}$ with the twin block compared to the activator. Moreover, it seems that twin block is able to better manage the inclination of the upper incisor with respect to the prespinal plane: compared to the $0.7^{\circ}$ reduction given by the Activator it has induced a $3.7^{\circ}$ reduction. Also the Ovj and Ovb dental parameters have undergone a greater improvement from twin block therapy (difference between the two devices, respectively, $-1.1 \mathrm{~mm}$ and $-0.2 \mathrm{~mm}$ ). On the other hand, analyzing the Andreasen activator device, it induced a more significant maxillomandibular divergence (SnaSnp-GoGn greater than $0.6^{\circ}$ in this group) and allowed to better control the inclination of the 
Table 3: Comparison in the results of the statistical analysis with unpaired $t$-test on cephalometric values of patients treated with Andreasen activator and twin block appliance

\begin{tabular}{|c|c|c|c|c|c|}
\hline \multicolumn{6}{|c|}{ Andreasen vs twin block } \\
\hline & Mean \pm SEM Andreasen & Mean \pm SEM Twin block & Difference & $p$ value & Significance \\
\hline SNA $\left({ }^{\circ}\right)$ & $-0.2 \pm 0.4422$ & $-0.2 \pm 0.2236$ & $0 \pm 0.4955$ & 0.9551 & NS \\
\hline SNB $\left(^{\circ}\right)$ & $2.1 \pm 0.4069$ & $2.3 \pm 0.2333$ & $0.2 \pm 0.469$ & 0.6999 & NS \\
\hline ANB $\left(^{\circ}\right)$ & $-2.3 \pm 0.5175$ & $-2.6 \pm 0.1633$ & $0.3 \pm 0.5426$ & 0.5916 & NS \\
\hline SnaSnp-GoGn $\left(^{\circ}\right)$ & $2.6 \pm 0.3712$ & $2 \pm 0.3333$ & $-0.6 \pm 0.4989$ & 0.2449 & NS \\
\hline Sna-Snp (mm) & $2.1 \pm 0.9597$ & $1.7 \pm 0.5588$ & $-0.4 \pm 1.111$ & 0.4828 & NS \\
\hline Co-Gn (mm) & $5.5 \pm 1.241$ & $5.9 \pm 0.9826$ & $0.4 \pm 1.583$ & 0.8035 & NS \\
\hline Co-Go (mm) & $4.5 \pm 0.9458$ & $4.3 \pm 0.9667$ & $-0.2 \pm 1.352$ & 0.5615 & NS \\
\hline Go-Gn (mm) & $3.9 \pm 0.7951$ & $3.7 \pm 1.202$ & $-0.2 \pm 1.91$ & 0.8914 & NS \\
\hline U1/SnaSnp $\left(^{\circ}\right)$ & $-0.7 \pm 1.126$ & $-3.7 \pm 1.086$ & $-3 \pm 1.564$ & 0.0711 & NS \\
\hline L1/GoGn $\left(^{\circ}\right)$ & $-0.4 \pm 0.8459$ & $1.2 \pm 1.093$ & $1.6 \pm 1.382$ & 0.2632 & NS \\
\hline Ovj (mm) & $-1.6 \pm 0.5207$ & $-2.7 \pm 0.7$ & $-1.1 \pm 0.8724$ & 0.2248 & NS \\
\hline $\mathrm{Ovb}(\mathrm{mm})$ & $-1.6 \pm 0.5164$ & $-1.8 \pm 0.5735$ & $-0.2 \pm 0.7717$ & 0.3138 & NS \\
\hline
\end{tabular}

${ }^{*} p<0.05 ;{ }^{* *} p<0.01 ;{ }^{* * *} p<0.001 ;{ }^{* * * *} p<0.0001 ;$ NS, not significant

lower incisor with respect to the mandibular plane. At the maxillary level, the decrease in the SnaSnp plane induced by this device was greater than $0.4 \mathrm{~mm}$ compared to the twin block. At the end of therapy, the Co-Go and Go-Gn parameters were slightly higher ( $+0.2 \mathrm{~mm}$ in both data) in patients treated with activator compared to those with twin block.

\section{Discussion}

Treatment with functional appliances should have hypothetically several advantages. In fact, functional appliance treatment can reduce overjet, improve patient's profile taking care of jaw discrepancies. It can also improve patient's self-esteem and minimize the risk of trauma to the upper incisors. ${ }^{18}$

The selection of functional appliances is dependent upon several factors which can be categorized into the patient factors, for example, age and compliance, and clinical factors, for example, preference/familiarity and laboratory facilities. ${ }^{9}$

At the maxillary level there are no statistically significant differences, with both types of devices we took into account. The SNA angle also decreased but to a very small extent in both groups. The length of the maxilla appears, instead, increased, at the end of therapy in all the treated patients. This can be explained by the continuation of the normal process of maxillary growth which occurs through a posterior bone neo-apposition and anterior reabsorption. As evidenced by the studies of Bhatia and Leighton, the amount of spontaneous growth of the maxilla is equal, in fact, to about $2 \mathrm{~mm} /$ year. ${ }^{19}$ The results obtained by the two devices took into consideration in our paper, in agreement with those present in the literature, ${ }^{20}$ do not identify, therefore, a real limitation in the growth of the maxillary but rather this is justifiable as a small and not significant headgear effect: they can stop the maxillary advancement minimally, an effect more than anything else due to the elastic reaction of the soft tissues generated by the mandibular advancement. $^{21}$

However, there are substantial differences at the lower jaw level. The SNB angle is increased: the functional devices studied have improved the sagittal relationship between the cranial base and the mandible, resulting in a significant anterior protrusion in the latter. This is absolutely consistent with the studies in the literature:
Tümer and Gültan, comparing the cephalometric effects produced by the Andreasen and the twin block, showed, in both cases, an improvement of $2^{\circ}$ of the same angle. ${ }^{22}$

In order to understand whether the devices studied only induced a mandibular repositioning or an effective growth, linear parameters were also analyzed. Co-Gn is, at the end of therapy, increased by $5.5 \mathrm{~mm}$ in the group treated with Andreasen and 5.9 $\mathrm{mm}$ in the group with twin block. Similar results are also present in the literature: ${ }^{23}$ the average increase in total mandibular growth obtained at the growth peak is about $5.5 \mathrm{~mm}$.

Referring to the literature, Stahl, Baccetti, Franchi and McNamara Jr. have showed that the discrepancy of mandibular growth between a class I and II, at the pubertal peak, is about 2 $\mathrm{mm}$ : the Co-Gn value in a class I, at the peak of growth, increased by $5.5 \mathrm{~mm}$ against the $3.5 \mathrm{~mm}$ of a II. ${ }^{24}$

Considering that $1 \mathrm{~mm}$ of deficit accumulates in the pre and post peak phase, the total mandibular growth deficit is $3 \mathrm{~mm}$ : therefore, to be able to say that a significant modification occurred was necessary to reach at least this value threshold. ${ }^{24}$ Because both devices made these effects, it can be assumed that there was a real additional mandibular growth in both experimental groups. The smallest increase $(+0.4 \mathrm{~mm})$ in the mandibular length induced by the twin block compared to the activator, which is highlighted in the present study, was also consistent with articles in the literature. ${ }^{22}$ The jaw, analyzed in its total length, cannot be increased without a simultaneous development of both mandibular ramus and body. In the present study, an increase of Co-Go equal to $4.6 \mathrm{~mm}$ and Go-Gn to $3.8 \mathrm{~mm}$ (average for both treated groups) was obtained at the end of the treatment. The slight difference, not statistically significant, of both parameters between the two devices is related to the minimum deviation in the total mandibular length induced and are consistent with those obtained by Mills and McCulloch. ${ }^{25}$ The growth of the mandibular ramus can be seen primarily as the effect, due to continuous protrusion, of induction of additional growth, through functional stimuli, on the main site of mandibular growth, the condyle, and secondarily as a response to vertical occlusal modification. ${ }^{26}$

The growth of the mandibular body (Go-Gn) makes a significant contribution to the total mandibular increase: it favors the forward displacement of the gonion and, consequently, is the main agent of the esthetic improvement, especially as regards the patient's profile. 
The significant growth and consequent sagittal progress obtained at the jaw level, together with the restraining effect on the maxilla, lead to a statistically and clinically significant reduction of the maxillomandibular skeletal relationship on the sagittal plane: the reduction of the ANB angle indicates the reduction of the severity of the malocclusion and is representative of the consequent improvement of the profile and facial esthetics. ${ }^{21}$

The interposition between the dental arches of resin coated guide surfaces, which act a bite, and the consequent downward and forward projection of the condyle, inducing the growth in the posterior direction, stimulate a clockwise rotation of the mandible (post-rotation): maxillomandibular divergence therefore increases. $^{27}$

It is important to remember that the effect just mentioned is also linked to the dentoalveolar modifications, like the desired extrusion of the posterior dental segments. Better and at the same time statistically significant results were obtained in this field by the activator: it can gain a more effective control of the vertical growth, as very often the patient to be able to perform the oral cavity functions, as phonation, free the contact with the occlusal plane. In the twin block, on the other hand, there is a greater dimensional control: its design support the classic bite blocks effect. ${ }^{20}$ This slight disparity, that not produce statistically significant effects between the two devices was also highlighted in the literature. ${ }^{22}$

The significant dentoalveolar effects of increased intermaxillary divergence and mandibular advancement are respectively reduction of overbite and overjet. About the latter parameter, the literature shows that $2 / 3$ of its decrease can be explained as skeletal consequences (mandibular repositioning) but $1 / 3$ to the induced dental ones. ${ }^{25}$ In fact, when a dental retention appliance is used, important reaction forces responsible for obvious effects are discharged onto the teeth. In the present study, the activator resulted in a slight and not statistically significant reduction of the upper and lower incisor inclination: this suggests that skeletal improvements were also useful to ameliorate this parameter. The group treated with twin block showed, in a statistically and clinically significant manner, an endo inclination of the upper incisors and a slight but not statistically significant proclination of the lower ones. These data reflect those cited in the literature. ${ }^{25,27}$

The advancement of the mandible translates into greater prominence of the chin and lower lip with consequent improvement of the relationships to the Ricketts E-line, increment of the labial-mental angle and reduction of the convexity of the profile. Also, the decrease of the overjet and, consequently, of the dental exposure increases usually result in an increase of patient's self-esteem. ${ }^{28}$

\section{ConcLusion}

The functional devices studied, Andreasen activator and twin block, seem to obtain excellent results, more skeletal than dental, if the treatment of II class malocclusion is made in patients at the peak of pubertal growth. At the end of the treatment, lower jaw appears advanced, with respect to the initial position, in relation to the cranial base and the upper jaw. Furthermore mandible appear increased in size at the level of the ramus, body and, consequently, in its total length.

In the upper jaw no significant changes were observed. Maxillomandibular relationship, analyzed in a sagittal projection appeared considerably improved not only skeletally but even in a dental view. It was also evident that part of the overjet was reduced by a dental compensation caused by an upper incisor retroclination and a lower proclination. Vertical dentoskeletal effects were clear: the increased maxillomandibular divergence, that is usually associated with these devices, was facilitated thanks to a selective extrusion of posterior teeth with consequent reduction of the overbite.

Both Andreasen activator and twin block have generated similar effects: for this reason it isn't possible privilege one of these devices. Both seem to be very effective and efficient. Quantitatively, the results have been remarkable, even if qualitatively they seem to require in many cases a finishing through a fixed orthodontic treatment, useful in making the occlusion more stable.

\section{References}

1. Maddalone $M$, Ferrari $M$, Stanizzi $A$, et al. Use of miniscrew implants in orthodontic distal movement [Utilizzo delle miniviti nelle meccaniche ortodontiche di distalizzazione]. Dent Cadmos 2010;78(8):97-105.

2. Maddalone $M$, Ferrari M, Barrila' $S$, et al. Intrusive mechanics in orthodontics with the use of TAD's. Dent Cadmos 2010;78(7): 97-106.

3. Maddalone M. The association between the psychological status and the severity of facial deformity in orthognathic patients. Angle Orthodontist 2012;82(3):396-402. DOI: 10.2319/060211-363.1.

4. Citterio F, Pellegatta A, Citterio $C L$, et al. Analysis of the apical constriction using micro-computed tomography and anatomical sections. G Ital Endod 2014;28(1):41-45. DOI: 10.1016/ j.gien.2014.05.001.

5. Venino PM, Citterio CL, Pellegatta $A$, et al. A micro-computed tomography evaluation of the shaping ability of two nickel-titanium instruments, HyFlex EDM and ProTaper next. J Endod 2017;43(4): 628-632. DOI: 10.1016/j.joen.2016.11.022.

6. Maddalone M, Gagliani M, Citterio $C L$, et al. Prevalence of vertical root fractures in teeth planned for apical surgery. A retrospective cohort study. Int Endod J 2018;51(9):969-974. DOI: 10.1111/iej.12910.

7. Maddalone M, Mirabelli L, Venino PM, et al. Long-term stability of autologous bone graft of intraoral origin after lateral sinus floor elevation with simultaneous implant placement. Clin Implant Dent Relat Res 2018;20(5):713-721. DOI: 10.1111/cid.12649.

8. Porcaro G, Busa A, Bianco E, et al. Use of a partial-thickness flap for guided bone regeneration in the upper jaw. J Contemp Dent Pract 2017;18(12):1117-1121. DOI: 10.5005/jp-journals-10024-2186.

9. Franchi L, Baccetti T. Prediction of individual mandibular changes induced by functional jaws orthopedics followed by fixed appliances in class II patients. Angle Orthod 2006;76(6):950-954. DOI: 10.2319/110205-385.

10. Cozza P, Baccetti T, Franchi L, et al. Mandibular changes produced by functional appliances in class II malocclusion: a systematic review. Am J Orthod Dentofacial Orthop 2006;129(5):599.e1-12. DOI: 10.1016/j. ajodo.2005.11.010.

11. Chen JY, Will LA, Niederman R. Analysis of efficacy of functional appliances on mandibular growth. Am J Orthod Dentofacial Orthop 2002;122(5):470-476. DOI: 10.1067/mod.2002.126730.

12. Manjusha KK, Jhothindrakumar K, Nishad A, et al. Growth and development of dentofacial complex influenced by genetic and environmental factors using monozygotic twins. J Contemp Dent Pract 2017;18(9):754-758. DOI: 10.5005/jp-journals-10024-2121.

13. Hughes T, Bockmann M, Mihailidis S, et al. Genetic, epigenetic, and environmental influences on dentofacial structures and oral health: ongoing studies of australian twins and their families. Twin Research and Human Genetics 2013;16(1):43-51. DOI: 10.1017/thg.2012.78.

14. Čirgić $\mathrm{E}$, Kjellberg $\mathrm{H}$, Hansen $\mathrm{K}$. Treatment of large overjet in angle class II: division 1 malocclusion with Andresen activators versus prefabricated functional appliances - a multicenter, randomized, controlled trial. Eur J Orthod 2016;38(5):516-524. DOI: 10.1093/ejo/ cjv080.

15. Isiekwe GI, DaCosta OO. Use of twin block of clark in management of angle's class II division I malocclusion. case report. NY State Dent J 2013;79(6):30-34. 
16. Mills CM, McCulloch KJ. Posttreatment changes after successful correction of class II malocclusions with the twin block appliance. Am J Orthod Dentofacial Orthop 2000;118(1):24-33. DOI: 10.1067/mod.2000.104902.

17. Baccetti T, Franchi L, McNamara JAJr. An improved version of the cervical vertebral maturation (CVM) method for the assessment of mandibular growth. Angle Orthod 2002;72(4):316-323.

18. O'Brien K, Wright J, Conboy F, et al. Effectiveness of treatment for class II malocclusion with the herbst or twin-block appliances: a randomized, controlled trial. Am J Orthod Dentofacial Orthop 2003;124(2):128-137. DOI: 10.1016/S0889-5406(03)00345-7.

19. Bhatia SN, Leighton BC. A manual of facial growth: a computer analysis of longitudinal cephalometric growth data. New York: Oxford University Press; 1993.

20. Lund DI, Sandler PJ. The effects of twin blocks: a Prospective controlled study. Am J Orthod Dentofacial Orthop 1998;113(1): 104-110. DOI: 10.1016/S0889-5406(98)70282-3.

21. Tarvade SM, Chaudhari CV, Daokar SG, et al. Dentoskeletal comparison of changes seen in class II cases treated by twin block and Forsus. $J$ Int Oral Health 2014;6(3):27-31.

22. Tümer N, Gültan AS. Comparison of the effects of monoblock and twin block appliances on the skeletal and dentoalveolar structures. Am J Orthod Dentofacial Orthop 1999;116(4):460-468. DOI: 10.1016/ S0889-5406(99)70233-7.
23. Pavoni C, Cretella Lombardo E, Franchi L, et al. Treatment and posttreatment effects of functional therapy on the sagittal pharyngeal dimensions in class II subjects. Int J Pediatr Otorhinolaryngol 2017;101:47-50. DOI: 10.1016/j.ijporl.2017.07.032.

24. Stahl F, Baccetti T, McNamara JAJr. Longitudinal growth changes in untreated subjects with class II division 1 malocclusion. Am J Orthod Dentofacial Orthop 2008;134(1):125-137. DOI: 10.1016/ j.ajodo.2006.06.028.

25. Mills CM, McCulloch KJ. Treatment effects of the twin block appliance: a cephalometric study. Am J Orthod Dentofacial Orthop 1998;114(1):15-24. DOI: 10.1016/S0889-5406(98)70232-X.

26. RufS, Pancherz H. Long term TMJ effects of herbst treatment: a clinical and MRI study. Am J Orthod Dentofacial Orthop 1998;114(5):475-483. DOI: 10.1016/S0889-5406(98)70166-0.

27. Perinetti G, Primozicc J, Franchi L, et al. Treatment effects of removable functional appliances in pre-pubertal and pubertal class II patients: a systematic review and meta-analysis of controlled studies. PLoS ONE 2015;10(10):e0141198. DOI: 10.1371/journal.pone. 0141198.

28. O'Brien K, Macfarlane T, Wright J, et al. Early treatment for class II malocclusion and perceived improvements in facial profile. Am J Orthod Dentofacial Orthop 2009;135(5):580-585. DOI: 10.1016/ j.ajodo.2008.02.020 